\author{
Ahi S. Issever \\ Thomas M. Link \\ Marie Kentenich \\ Patrik Rogalla \\ Andrew J. Burghardt \\ Galateia J. Kazakia \\ Sharmila Majumdar \\ Gerd Diederichs
}

\section{Assessment of trabecular bone structure using MDCT: comparison of 64- and 320-slice CT using HR-pQCT as the reference standard}

Received: 18 January 2009

Revised: 24 June 2009

Accepted: 5 July 2009

Published online: 27 August 2009

(C) The Author(s) 2009. This article is published with open access at Springerlink.com

\author{
A. S. Issever - M. Kentenich · \\ P. Rogalla $\cdot$ G. Diederichs \\ Department of Radiology, Charité \\ Campus Mitte, Universitaetsmedizin, \\ Berlin, Germany
}

\author{
A. S. Issever · T. M. Link · \\ M. Kentenich - A. J. Burghardt . \\ G. J. Kazakia - S. Majumdar • \\ G. Diederichs \\ Department of Radiology and \\ Biomedical Imaging, Musculoskeletal \\ and Quantitative Imaging Research \\ Group, University of California, \\ San Francisco, CA, USA
}

\author{
A. S. Issever ( $\bowtie)$ \\ Department of Radiology, \\ Charité Campus Mitte, \\ Universitaetsmedizin Berlin, \\ Charitéplatz 1, \\ 10117 Berlin, Germany \\ e-mail: ahi-sema.issever@charite.de \\ Tel.: +49-30-627043 \\ Fax: +49-30-527910
}

\begin{abstract}
Objectives: The aim of our study was to perform trabecular bone structure analysis with images from 64- and 320-slice multidetector computed tomography (MDCT) and to compare these with high-resolution peripheral computed tomography (HR-pQCT). Materials and methods: Twenty human cadaver distal forearm specimens were imaged on a 64- and 320-slice MDCT system at $120 \mathrm{kVp}, 200 \mathrm{~mA}$ and $135 \mathrm{kVp}$, $400 \mathrm{~mA}$ (in-plane pixel size $234 \mu \mathrm{m}$; slice thickness $500 \mu \mathrm{m})$. HR-pQCT
\end{abstract}

imaging was performed at an isotropic voxel size of $41 \mu \mathrm{m}$. Bone volume fraction (BV/TV), trabecular number (Tb.N), thickness (Tb.Th) and separation (Tb.Sp) were computed. Results: MDCT-derived BV/TV and $\mathrm{Tb} . \mathrm{Sp}$ were highly correlated $(r=0.92-0.96, p<0.0001)$ with the corresponding HR-pQCT parameters. $\mathrm{Tb}$.Th was the only structure measure that did not yield any significant correlation. Conclusion: The 64and 320-slice MDCT systems both perform equally well in depicting trabecular bone architecture. However, because of constrained resolutions accurate derivation of trabecular bone measures is limited to only a subset of microarchitectural parameters.

Keywords 320-slice MDCT . HR-pQCT · Osteoporosis · Structure analysis - Trabecular bone

\section{Introduction}

Osteoporosis is defined as a systemic skeletal disease characterised by a loss of bone mass and a deterioration of bone microarchitecture, subsequently leading to increased susceptibility to fracture [1]. Numerous studies have shown that bone mineral density (BMD) is a fundamental determinant of bone strength [2-7]. Hence, the established method in current osteoporosis diagnosis is the assessment of BMD using either dual-energy X-ray absorptiometry (DXA) or quantitative computed tomography (QCT). However, clinicians and researchers have learned in recent years that BMD only accounts for approximately $60-70 \%$ of bone strength [1, 8], and sufficient discrimination between patients with and without increased fracture risk is not provided by BMD measurements alone [9-11]. The National Institutes of Health Consensus Conference on Osteoporosis introduced the concept of "bone quality" in 2001, referring to all characteristics of bone-apart from BMD - responsible for its strength [1]. Among these characteristics trabecular bone structure has been identified as a major contributor to bone strength [12-14].

With average trabecular dimensions ranging from 50 to $200 \mu \mathrm{m}$, depiction and analysis of trabecular bone require high-resolution imaging techniques. Micro-computed tomography $(\mu \mathrm{CT})$, which is capable of approximately 3 - 
$\mu \mathrm{m}$ isotropic resolutions, may currently be considered the most established high-resolution imaging technique for trabecular bone depiction. However, the application of $\mu \mathrm{CT}$ imaging is restricted to either in vitro samples or invasive biopsy imaging and is therefore not suitable for daily clinical use. In the field of in vivo trabecular bone imaging, multidetector computed tomography (MDCT) and high-resolution magnetic resonance imaging (HRMRI) have both been investigated in detail, but with inplane pixel sizes of approximately $100-300 \mu \mathrm{m}$ and slice thicknesses of $300-500 \mu \mathrm{m}$ resolution constraints remain [15-18].

Recently, a high-resolution peripheral quantitative computed tomography (HR-pQCT) device was introduced that permits in vivo imaging of distal sites at a resolution of less than $100 \mu \mathrm{m}$ [19]. MacNeil and Boyd tested the accuracy of this HR-pQCT device - at an isotropic $82-\mu \mathrm{m}$ voxel size on ten distal radii specimens using $\mu \mathrm{CT}$ (isotropic $19-\mu \mathrm{m}$ voxels) as the standard [20]. Highly significant correlations for Tb.N were found $\left(R^{2}=0.95\right)$. HR-pQCT measures of Tb. Th showed the lowest correlation $\left(R^{2}=0.59\right)$ with $\mu \mathrm{CT}$ in this study. This suggests that HR-pQCT may be used as a standard of reference for in vivo trabecular bone imaging of the distal radius and the distal tibia.

However, as the availability of HR-pQCT systems is limited to only a few specialised facilities, implementation into research and clinical practice may be considered restricted and strategies involving more accessible techniques such as MDCT and HR-MRI for in vivo trabecular bone imaging will remain essential in future osteoporosis management. The current study was performed to evaluate the feasibility of MDCT-derived trabecular bone structure analysis on intact human cadaver forearm specimens. The aim of this study was to compare MDCT-derived apparent structure measures with HR-pQCT-derived structure measures as the standard of reference, and to evaluate possible differences in the derivation of apparent structure measures using two different MDCT devices (64-slice vs. 320-slice) with two different protocols $(120 \mathrm{kVp}$ and $200 \mathrm{~mA}$ vs. $135 \mathrm{kVp}$ and $400 \mathrm{~mA}$ ).

\section{Materials and methods}

\section{Specimens}

Twenty human cadaver forearms fixed in $4 \%$ paraformaldehyde were obtained from ten body donors (seven women, three men; mean age 82 years, range 6191 years). The specimens were obtained from the Department of Anatomy, Charité Campus Mitte, Universitaetsmedizin Berlin, Germany. Before death, all donors had given signed consent to dedicate their bodies to research. The forearms were removed from the remaining upper extremity distal from the elbow joint. Criteria for exclusion from the study were evidence of previous fracture, osteolytic or osteoblastic bone lesions, and high-grade osteoarthritis.

Multidetector computed tomography (MDCT) imaging

MDCT examinations of the whole specimens were performed using 64-slice (Toshiba Aquilion 64, Otawara, Japan) and 320-slice MDCT systems (Toshiba Aquillion ONE, Otawara, Japan) with the following protocols: (A) standard dose protocol with a tube current of $120 \mathrm{kVp}$ and $200 \mathrm{~mA}$ (rotation time $0.5 \mathrm{~s}$ ), and (B) high-dose protocol with $135 \mathrm{kVp}$ and $400 \mathrm{~mA}$ (rotation time $1.0 \mathrm{~s}$ ). The acquisition mode on the 64-slice MDCT device was a helical CT mode, whereas a volume CT mode was used for the 320-slice MDCT device. A slice thickness of $0.5 \mathrm{~mm}$ was obtained in both protocols and devices. The reconstruction interval was set to $0.3 \mathrm{~mm}$ on the 64-slice CT system and to $0.25 \mathrm{~mm}$ on the 320 -slice CT system. Images were reconstructed with a field of view (FOV) of $120 \mathrm{~mm}$ on a standard image matrix size of $512 \times 512$ pixels yielding an in-plane pixel size of $0.234 \mathrm{~mm}$ (Table 1). The imaging range extended from the metacarpophalangeal joints to the mid third of the radius. A high-resolution kernel (FC 81) was used for postprocessing of the 64-slice MDCT images. As this kernel option was not available for the 320-slice MDCT a standard bone kernel (FC 30) in combination with an edge-enhancing filter was used on the 320-slice MDCT images. For each scan protocol the manufacturer's modulation transfer function (MTF) values for the 64- and 320slice MDCT devices are given in Table 1. Consistent with the standard clinical patient scanning protocol (subject positioned prone with the arm extended above the head) the specimens were placed supine (palmar side down) and along the axis of the forearm on the $\mathrm{CT}$ table. In addition to assess BMD a standard hydroxyapatite (HA)-based calibration phantom - consisting of five tubular inserts with varying concentrations $\left(0,50,100,150,200 \mathrm{mg} \mathrm{HA} / \mathrm{cm}^{3}\right)$-was placed underneath the specimens.

High-resolution peripheral quantitative computed tomography (HR-pQCT)

After MDCT imaging all specimens underwent imaging with a clinical HR-pQCT system (XtremeCT, Scanco Medical AG, Bassersdorf, Switzerland). To ensure optimal image co-registration the specimens were positioned in the same manner as they were positioned for the MDCT (supine and along the axis of the forearm). A modified standard in vivo protocol at the distal end of the radius was used. The X-ray source potential was set to $60 \mathrm{kVp}$ with a current of $900 \mu \mathrm{A}$. The $126-\mathrm{mm}$ field of view (FOV) was reconstructed across a $3,072 \times 3,072$ matrix, yielding 41 $\mu \mathrm{m}$ isotropic voxels. 
Table 1 Imaging protocols and specifics applied with regard to 64- and 320-slice MDCT

\begin{tabular}{lllll}
\hline MDCT (slice) & 320 & & \multicolumn{2}{l}{ Helical } \\
\cline { 2 - 4 } CT mode & Volume & & A & B \\
\hline Protocol & $\mathrm{A}$ & $\mathrm{B}$ & 120 & 135 \\
$\mathrm{kVp}$ & 120 & 135 & 200 & 400 \\
$\mathrm{~mA}$ & 200 & 400 & 0.5 & 1.0 \\
Imaging time $(\mathrm{s})$ & 0.5 & 1.0 & $<0.1$ & $<0.1$ \\
Radiation dose $(\mathrm{mSV})$ & $<0.1$ & $<0.1$ & FC 81 (high-resolution) \\
Kernel & FC 30 (bone) + edge enhancing & 11.33 & 9.00 \\
MTF 50\% & 9.41 & 7.46 & 13.27 & 12.52 \\
MTF 10\% & 11.76 & 9.86 & 234 & 234 \\
In-plane pixel size $(\mu \mathrm{m})$ & 234 & 234 & 500 & 500 \\
Slice thickness $(\mu \mathrm{m})$ & 500 & 500 & 300 & 300 \\
Slice increment $(\mu \mathrm{m})$ & 250 & 250 &
\end{tabular}

Concerning the radiation dose please note that the radiation dose for the standard HR-pQCT scans, which are performed at an isotropic resolution of $82 \mu \mathrm{m}^{3}$, is below $0.3 \mu \mathrm{SV}$

Based on the two-dimensional X-ray scout view, the image was defined to begin $9.5 \mathrm{~mm}$ proximal to the midline of the distal radius endplate (Fig. 1). A three-dimensional (3D) stack of 220 high-resolution CT slices over a 9.02mm-thick section was acquired. The overall imaging time was $7.4 \mathrm{~min}$ per specimen. The calibration of the reconstructed linear attenuation values was carried out using a separate density measurement performed on a hydroxyapatite phantom before imaging.

\section{ROIs and image registration}

The regions of interest (ROI) for the evaluation of structural parameters were placed in the trabecular compartment of the distal radius, along the cortical and

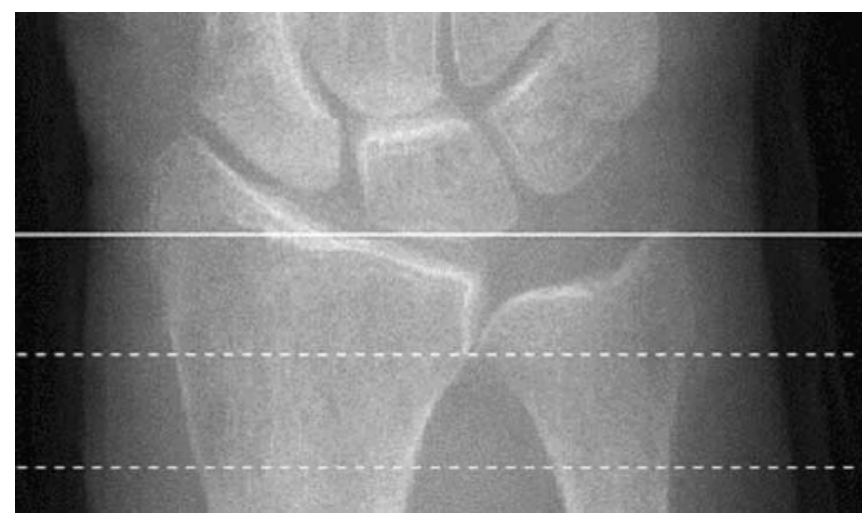

Fig. 1 HR-pQCT scout view. The imaging procedure was defined to begin $9.5 \mathrm{~mm}$ proximal to the midline of the distal radius endplate (white line). The imaging range covered $9.0 \mathrm{~mm}$ (distance between the dotted lines) trabecular bone interface, with an internal, endosteal offset of at least $1 \mathrm{~mm}$. Visual registration was used to evaluate images of the same anatomical site on the HR-pQCT, 320slice MDCT and 64-slice MDCT images. On the basis of the standardised specimen positioning during each procedure, similar axial slices were identified in each of the three imaging datasets and defined as starting slices for further evaluation. Characteristics of the trabecular bone network and the surrounding cortex served as confirmation (Fig. 2 $\mathrm{a}, \mathrm{c}$ and e). To cover the same imaging range of $9.0 \mathrm{~mm}$ as used in HR-pQCT imaging, 30 images were evaluated in the 64-slice MDCT datasets (in increments of $300 \mu \mathrm{m}$ ) and 36 axial images in the 320-slice MDCT datasets (in increments of $250 \mu \mathrm{m}$ ).

\section{Trabecular structure analysis}

Structural analysis necessitates segmentation of grey-level images into binarised images consisting only of a bone phase and a bone marrow phase (Fig. 2 b, d and f). However, as the histogram characteristics of HR-pQCT (bimodal) and MDCT (monomodal) vary, different thresholding techniques have to be applied. Furthermore, because of differences in image characteristics and spatial resolution $\left(41 \times 41 \times 41 \mu^{3}\right.$ isotropic voxels in HR-pQCT vs. $234 \times 234 \times 300 / 250 \mu^{3}$ anisotropic voxels in MDCT) different algorithms to compute structural parameters have to be used for each technique.

$H R-p Q C T$ Structure analysis of the HR-pQCT images was performed using the software provided by the manufacturer. Image binarisation was performed within the defined trabecular ROI after applying a global threshold. The global threshold was calculated for each specimen using a 
Fig. 2 Cross-sectional HRpQCT image at an isotropic 41$\mu \mathrm{m}$ resolution (a). Matched MDCT images (protocol B) after visual image co-registration acquired with the 64-slice (c) and 320-slice (e) MDCT systems. Subfigures b, $\mathbf{d}$ and $\mathbf{f}$ show the corresponding binarised images
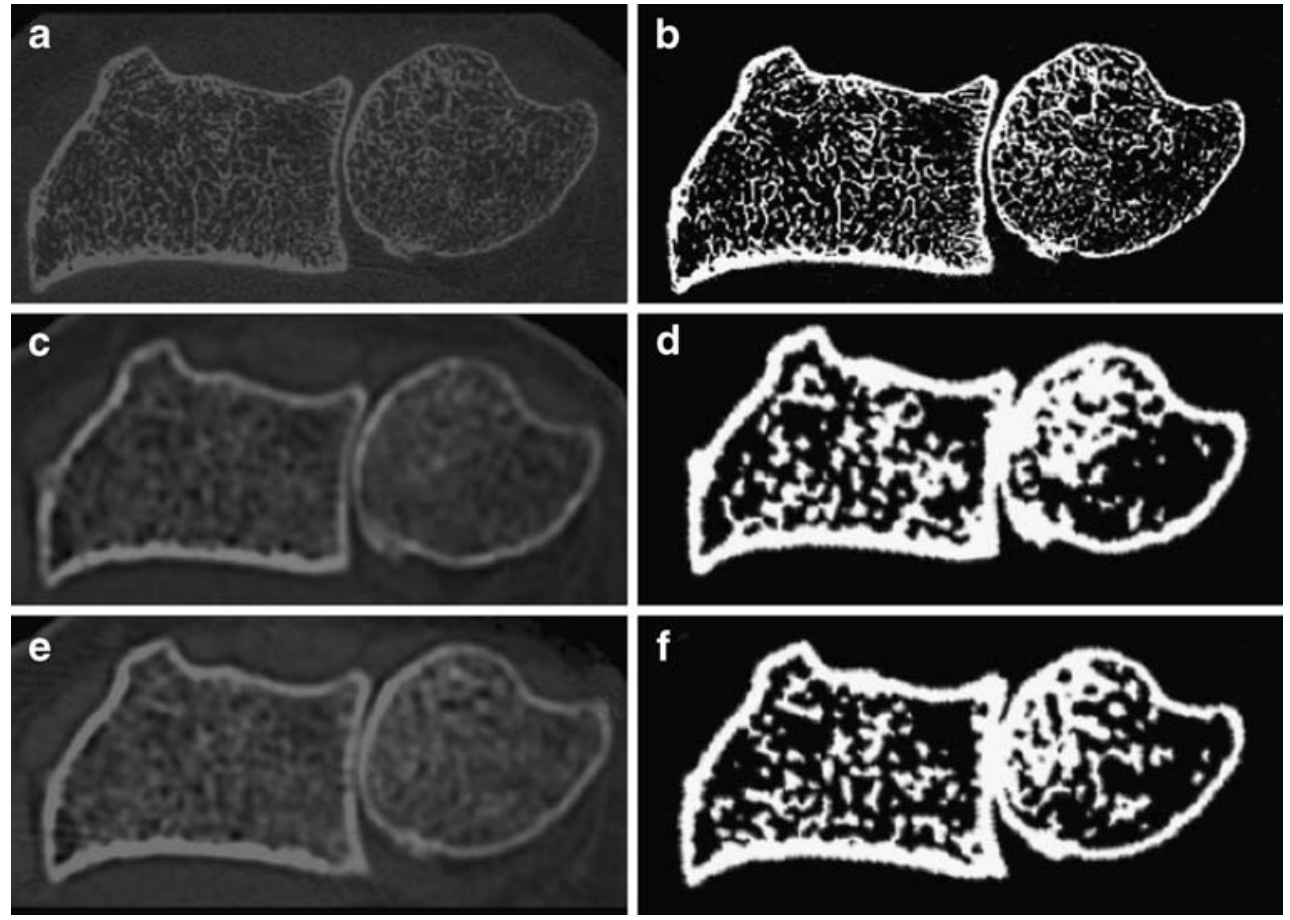

common adaptive iterative method upon a specific specimen-based histogram analysis [21]. The underlying assumption with regard to this method is that the histogram intensity distribution is bimodal, expressing a bone and background peak. The midpoint between the two peaks was used as the threshold value. After image binarisation bone volume/total volume (BV/TV) was derived via simple voxel counting. Calculation of trabecular number (Tb.N), trabecular thickness (Tb.Th) and trabecular separation (Tb.Sp) was performed without model assumptions as direct measures [22]. Based on the assumption that compact bone has an HA density value of $1,200 \mathrm{mg} / \mathrm{cm}^{3}$, BMD of the trabecular compartment was obtained after calibration against a phantom composed of five cylinders of HA-resin mixtures with a range of mineral concentrations $(0,100,200,400$, $800 \mathrm{mg} \mathrm{HA} / \mathrm{cm}^{3}$ ). Overall HR-pQCT measures served as the standard of reference in this study.

MDCT Structure analysis of the MDCT images was performed using in-house-developed image analysis software programmed in IDL (RSI, Boulder, CO, USA). For threshold definition purposes six different thresholds based on six different HA density values $(50,100,150$, 200,250 and $300 \mathrm{mg} \mathrm{HA} / \mathrm{cm}^{3}$ ) were applied to each MDCT dataset. The HA density values were reconverted to Hounsfield units (HU) via back-calibration using the manufacturer's phantom on which the specimens were placed during the examination. The inverse calculation (HU to HA) was used to measure the BMD of the trabecular compartment. Structural measures obtained for each MDCT dataset with each of the six thresholds were compared with the standard structure measures as assessed with the HR-pQCT. The overall global threshold was defined to be at the HA density level that computed an optimal combination of correlations, as described later. Following image binarisation apparent trabecular structure measures were derived, as previously described in detail by Majumdar and Genant [23]. Structural parameters analogous to those obtained with standard histomorphometry techniques were assessed, but defined to be apparent (app.) structure measures, as standard histomorphometry analysis is performed at much higher spatial resolutions (voxel sizes, approximately $5 \mu \mathrm{m}$ ). The following apparent parameters were obtained: app. BV/TV, app. Tb.N, app. Tb.Th and app. Tb.Sp. Briefly, the total number of bright pixels contributing to the bone phase in the binarised image relative to the total number of pixels in the ROI was used to compute app. BV/TV. The total number of black and white pixel edges that cross a set of parallel rays at a given angle $\theta$ through the image were counted, then a measure of the mean intercept length was computed as the ratio between the total area of the bright pixels and half the number of edges. The mean value of the intercept length for all angles provided the width of the bright pixels and was defined as apparent trabecular thickness. From these measurements of app. BV/TV and app. Tb.Th, two other morphological parameters were determined: app. Tb.N (area fraction of bright pixels/app. Tb.Th) and app. Tb.Sp ([1/app. Tb.N]-app. Tb.Th). 


\section{Statistics}

Mean values and standard deviations were calculated for HR-pQCT-derived BV/TV, Tb.N, Tb.Th, Tb.Sp and BMD measures and for MDCT-derived app. BV/TV, app. Tb.N, app. Tb.Th, app. Tb.Sp and BMD measures. Furthermore two-sided 95\% confidence intervals were computed. Oneway analysis of variance (ANOVA) tests were applied to calculate differences in the mean values. Using linear regression analysis, Spearman rank's correlation coefficient $r$ and the coefficient of determination $R^{2}$ were obtained to compare parameters. The level of significance was set at $p<0.05$, and the level of high significance was set at $p<0.0001$. All calculations were performed with JMP software (SAS Institute, Cary, NC, USA).

\section{Results}

\section{HR-pQCT vs. MDCT density measures}

Highly significant correlations with $r=0.97$ to $r=0.99(p<$ 0.0001) were found among BMD values of the trabecular compartment as assessed by HR-pQCT- and all MDCTderived BMD values. Student's $t$ test showed that the density measures showed no statistically relevant difference $(p>0.05)$ in the values acquired. The mean density value as assessed with all techniques and imaging protocols was $125.63 \pm 58.48 \mathrm{mg} \mathrm{HA} / \mathrm{cm}^{3}$.

Highly significant $(p<0.0001)$ correlations were found for HR-pQCT BMD measures vs. BV/TV $(r=0.96)$, Tb.Sp $(r=-0.89)$, and Tb.N $(r=0.87)$. Tb.Th was the only measure that showed no significant correlation with BMD.

\section{Threshold definition in the MDCT datasets}

To define the optimal global threshold, significant $(p<$ 0.05 ) Spearman correlation coefficient $r$ values (MDCT vs. HR-pQCT) — for all structure measures - were evaluated as a function of the applied threshold level $(50,100,150,200$,
250 and $300 \mathrm{mg} \mathrm{HA} / \mathrm{cm}^{3}$ ). Overall, the highest and most significant correlation was found for BV/TV, but with increasing threshold - especially for measures acquired using protocol $\mathrm{A}-\mathrm{a}$ gradual decrease in the correlation was observed (e.g. 64-slice $\mathrm{MDCT}_{\text {protocol }}$ A from $r_{50 \mathrm{mg} \mathrm{HA} / \mathrm{cm}^{3}}=0.98$ to $\left.r_{300 \mathrm{mg} \mathrm{HA} / \mathrm{cm}^{3}}=0.77\right)$. A decreasing correlation with increasing threshold was also observed for Tb.Sp (64-slice MDCT $\mathrm{Mrotocol} \mathrm{A}_{\text {A }}$ from $r_{50 \mathrm{mg} \mathrm{HA} / \mathrm{cm}^{3}}=0.95$ to $\left.r_{200 \mathrm{mg} \mathrm{HA} / \mathrm{cm}^{3}}=0.47\right)$. Depending on the device type and protocol the correlations for Tb.Sp lost significance $(p>$ 0.05 ) after applying a threshold of 150 to $250 \mathrm{mg} \mathrm{HA} / \mathrm{cm}^{3}$. Inversely for correlations of Tb.N, significance was only achieved above a threshold level of $150 \mathrm{mg} \mathrm{HA} / \mathrm{cm}^{3}$, whereas for $\mathrm{Tb}$.Th significance was only found within the threshold level range of at least 200 to $300 \mathrm{mg} \mathrm{HA} / \mathrm{cm}^{3}$. Thus - as shown in Fig. 3 (320-slice $\mathrm{MDCT}_{\text {protocol A}}$ ), for example - the optimal combination of correlations was found - for each protocol, and each device - to be the $150 \mathrm{mg} \mathrm{HA} / \mathrm{cm}^{3}$ density level, which was then defined as the global threshold for further evaluation.

\section{HR-pQCT vs. MDCT structure measures}

Table 2 shows HR-pQCT- and MDCT-derived mean and standard deviation values for BV/TV, Tb.N, Tb.Th and Tb.Sp. Compared with HR-pQCT-derived structure measures, MDCT-derived measures yielded two- to threefold higher values for app. Tb.Th, up to twofold higher values for app. Tb. $\mathrm{Sp}$, and increased app. BV/TV measures, thus overall resulting in a smaller amount of trabeculae detected, as seen by nearly $50 \%$ decreased app. Tb.N values.

ANOVA showed no device- (64-slice vs. 320-slice) or protocol-dependent difference $(120 \mathrm{kVp}$ and $200 \mathrm{~mA}$ vs. $135 \mathrm{kVp}$ and $400 \mathrm{~mA}$ ) in the primary apparent structure measures (app. BV/TV and app. Tb.Th) with $p$ values above 0.2. When comparing HR-pQCT-derived structure measures with MDCT-derived apparent structure measures (Table 3$)$ highly significant correlations $(p<0.0001)$ were found for BV/TV and Tb.Sp with $r$ values of 0.95 to 0.96
Fig. 3 Variations in the correlations between structure measures derived from 320 -slice MDCT using protocol A compared with HR-pQCT-derived structure measures are presented as a function of the applied threshold. The optimal combination of correlations is found at the level of $150 \mathrm{mg} \mathrm{HA} / \mathrm{cm}^{3}$

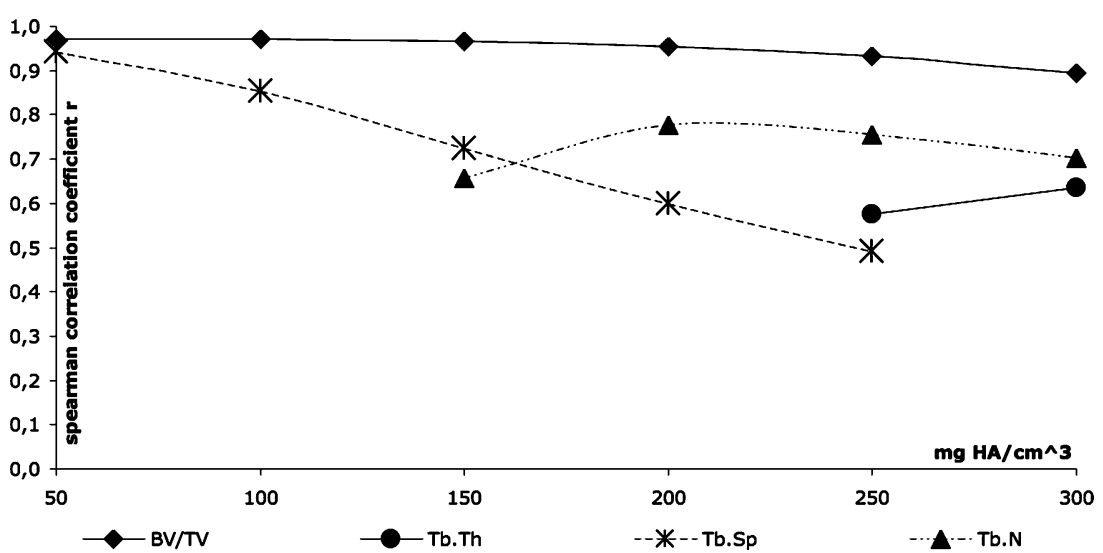


Table 2 HR-pQCT-derived trabecular structure measures and MDCT-derived apparent trabecular structure measures acquired with the 64slice (64) and the 320-slice (320) MDCT device

\begin{tabular}{llllll}
\hline & HR-pQCT & MDCT & & & \\
\cline { 2 - 6 } & & $64-\mathrm{A}$ & $320-\mathrm{A}$ & $64-\mathrm{B}$ & $320-\mathrm{B}$ \\
\hline BV/TV $(\%)$ & $24 \pm 6[22 ; 27]$ & $39 \pm 19[30 ; 48]$ & $46 \pm 17[37 ; 54]$ & $40 \pm 21[29 ; 50]$ & $36 \pm 19[27 ; 45]$ \\
Tb.N (1/mm) & $1.38 \pm 0.30[1.24 ; 1.52]$ & $0.56 \pm 0.15[0.49 ; 0.63]$ & $0.68 \pm 0.12[0.63 ; 0.73]$ & $0.47 \pm 0.14[0.41 ; 0.54]$ & $0.50 \pm 0.16[0.42 ; 0.58]$ \\
Tb.Th (mm) & $0.25 \pm 0.02[0.24 ; 0.26]$ & $0.67 \pm 0.22[0.56 ; 0.78]$ & $0.65 \pm 0.20[0.56 ; 0.75]$ & $0.79 \pm 0.31[0.64 ; 0.94]$ & $0.68 \pm 0.22[0.58 ; 0.78]$ \\
Tb.Sp (mm) & $0.77 \pm 0.31[0.63 ; 0.92]$ & $1.31 \pm 0.87[0.90 ; 1.73]$ & $0.87 \pm 0.46[0.65 ; 1.09]$ & $1.62 \pm 1.20[1.04 ; 2.19]$ & $1.66 \pm 1.21[1.09 ; 2.23]$ \\
\hline
\end{tabular}

Data are expressed in mean \pm standard deviation, $95 \%$ confidence interval [lower bound, upper bound]

$A 120 \mathrm{kVp}, 200 \mathrm{~mA} ; B 135 \mathrm{kVp}, 400 \mathrm{~mA}$

and 0.92 to 0.93 , respectively. Tb.N showed weaker correlations; nonetheless, significant $r$ values of 0.55 to highly significant $r$ values of 0.82 were obtained for the 320 -slice MDCT with protocol B. Neither of the MDCT devices (64 vs. 320) nor protocols (A vs. B) produced significant correlations for $\mathrm{Tb}$. Th values. When comparing the MDCT-derived apparent structure measures among each other (320 vs. 64 and protocol A vs. B) highly ( $p<$ $0.0001)$ significant correlations are found as shown in Figs. 4 and 5, with the lowest protocol-dependent $R^{2}$ of 0.84 for app. Tb.N as assessed with the 320 -slice MDCT device.

\section{Discussion}

In this study trabecular bone structure measures acquired with two different MDCT devices and two different protocols were compared with HR-pQCT. The results show that most MDCT-derived structure measures yield highly significant correlations to HR-pQCT. Furthermore, no MDCT device- (320-slice vs. 64-slice) or protocoldependent (120 kVp and $200 \mathrm{~mA}$ vs. $135 \mathrm{kVp}$ and $400 \mathrm{~mA})$ differences were identified for primary trabecular structure measures.

The current, clinically established method of diagnosing osteoporosis is the assessment of BMD. BMD accounts for approximately $60-70 \%$ of bone strength $[1,8]$; however, for the purposes of fracture risk discrimination the assessment of BMD alone is insufficient. There is a substantial overlap of patients with manifest osteoporotic fractures and normal BMD values vs. patients without fractures and osteoporotic BMD values [10, 25].

The term "bone quality" was introduced by the National Institutes of Health Consensus Conference on Osteoporosis in 2001, encompassing all characteristics and features of bone responsible for its strength, such as tissue composition, geometry, turnover, cortical and trabecular microarchitecture, pre-existing damage accumulation etc. [1]. As bone turnover processes are highly surface-sensitive, trabecular bone microarchitecture is apt to reflect physiological, pathophysiological and drug-induced changes. Thus, many studies have focused on the quantitative analysis of trabecular bone. Hildebrand et al. found that a combination of BMD and trabecular structure measures may explain $94 \%$ of trabecular bone strength compared with $64 \%$ if only BMD measures are used [22]. The trabecular bone structure of specimens from patients with fractures, as compared with those without fractures, is characterised by a significantly higher degree of anisotropy, with proportionately fewer trabecular elements transverse to the primary load axis [26]. In a histomorphometric study of transiliac bone biopsies of 152 men it was revealed that the number of clinical risk factors for osteoporosis is strongly related to microarchitectural alterations of trabecular bone [27]. The assessment and analysis of trabecular

Table 3 Correlation coefficients (Spearman) of MDCT-derived apparent trabecular structure measures compared with HR-pQCT-derived trabecular structure measures

\begin{tabular}{|c|c|c|c|c|}
\hline \multirow[t]{2}{*}{ Structural parameters } & \multicolumn{4}{|c|}{ MDCT vs. HR-pQCT } \\
\hline & $64-\mathrm{A}$ & $64-\mathrm{B}$ & $320-\mathrm{A}$ & $320-\mathrm{B}$ \\
\hline $\mathrm{BV} / \mathrm{TV}$ & $0.96^{*}$ & $0.95^{*}$ & $0.96^{*}$ & $0.95^{*}$ \\
\hline Tb.N & $0.76^{* *}$ & $0.65^{* *}$ & $0.55^{* *}$ & $0.82^{*}$ \\
\hline Tb.Th & NS. & NS & NS & NS \\
\hline $\mathrm{Tb} . \mathrm{Sp}$ & $0.93^{*}$ & $0.93^{*}$ & $0.93^{*}$ & $0.92^{*}$ \\
\hline
\end{tabular}

NS nonsignificant correlations are not listed ${ }^{*} p<0.0001 ;{ }^{* *} p<0.05$ 


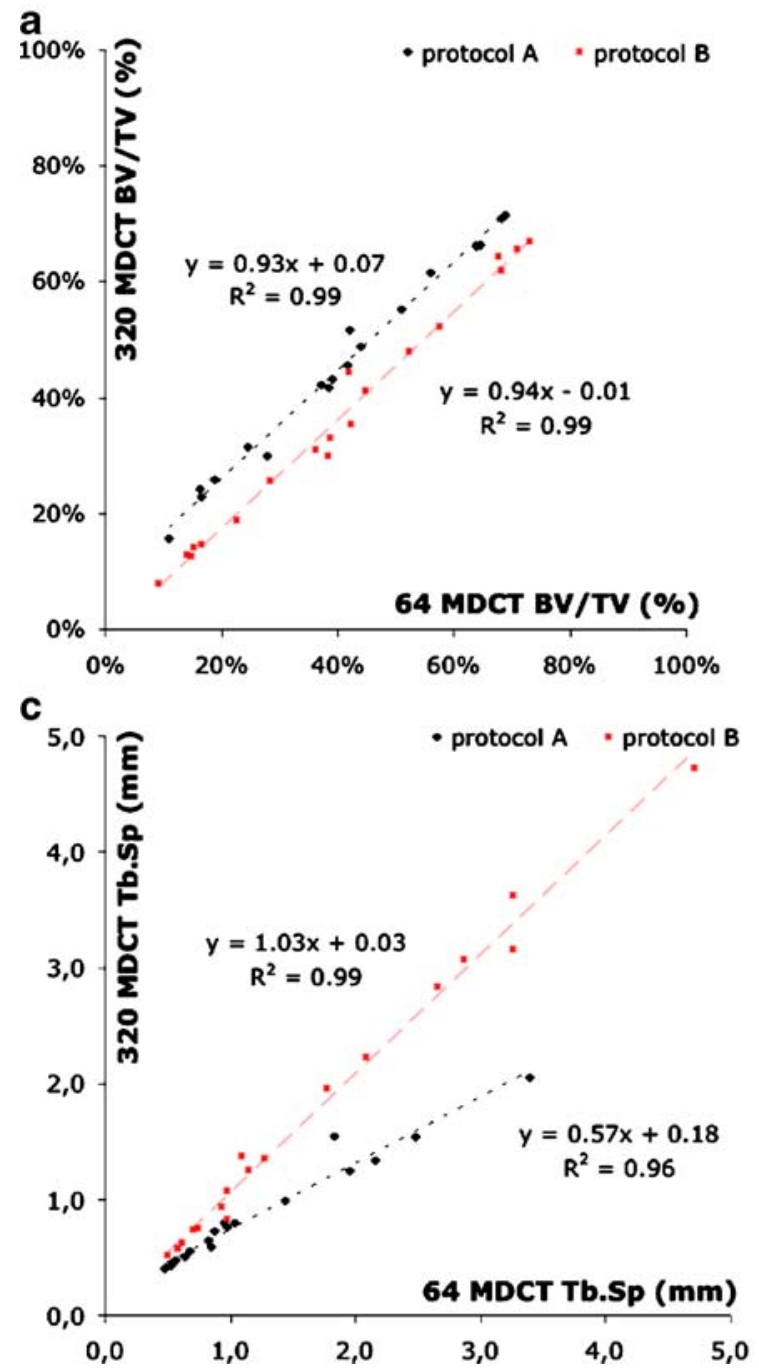

Fig. 4 Comparison of apparent trabecular structure measures (BV/ TV (a), Tb.N (b), Tb.Sp (c), Tb.Th (d)) derived using 320-slice vs. 64-slice MDCT as assessed with protocol A (120 kVp and $200 \mathrm{~mA}$,

microarchitecture, in combination with BMD measures, may thus provide substantial advancement in the field of osteoporosis diagnosis and therapy monitoring. Therefore, in daily clinical practice noninvasive - ideally high-resolution-imaging techniques, capable of depicting trabecular bone, are needed.

The first in vivo studies investigating trabecular bone structure assessment were performed nearly 20 years ago, and since then there have been significant technical advances improving the spatial resolution of imaging [28, 29]. The first peripheral quantitative computed tomography devices were limited to nominal isotropic resolutions of $170 \mu \mathrm{m}[30]$, but with the HR-pQCT systems, in vivo imaging at a nominal isotropic resolution of $82 \mu \mathrm{m}$ is now applicable at distal sites [19]. A number of studies have already been published with HR-pQCT as the primary

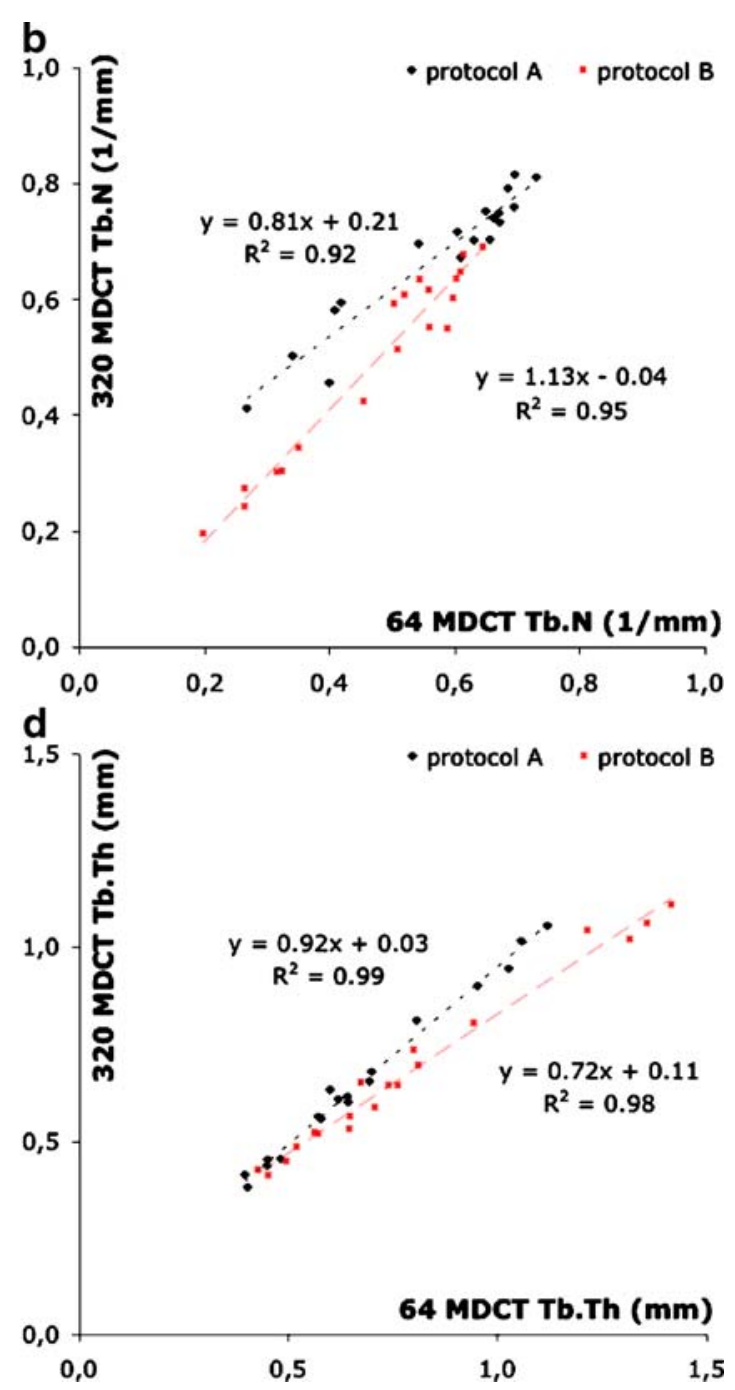

black dots $)$ and protocol $\mathrm{B}(135 \mathrm{kVp}$ and $400 \mathrm{~mA}$, red dots $)$. Coefficient of determination $R^{2}$ is given

imaging technique for trabecular bone structure analysis $[19,24,31,32]$.

The underlying motive of the current study was to evaluate the in vivo feasibility of trabecular bone structure analysis at the distal radius, a commonly affected osteoporosis fracture site. The lifetime risk of women at the age of 50 of sustaining a wrist fracture ranges from $13.3 \%$ in Australia to $20.8 \%$ in Sweden [33]. A recently published study has identified that previous wrist fractures strongly predict the 3-year risk of any future osteoporotic fracture in postmenopausal women, independent of BMD [34]. Among the few published HR-pQCT studies, Kazakia et al. and Krug et al. compared HR-MRI- and HR-pQCTderived in vivo structure measures of the distal radius [3537]. They found highly significant intertechnique correlations; however, it was also observed that there are 


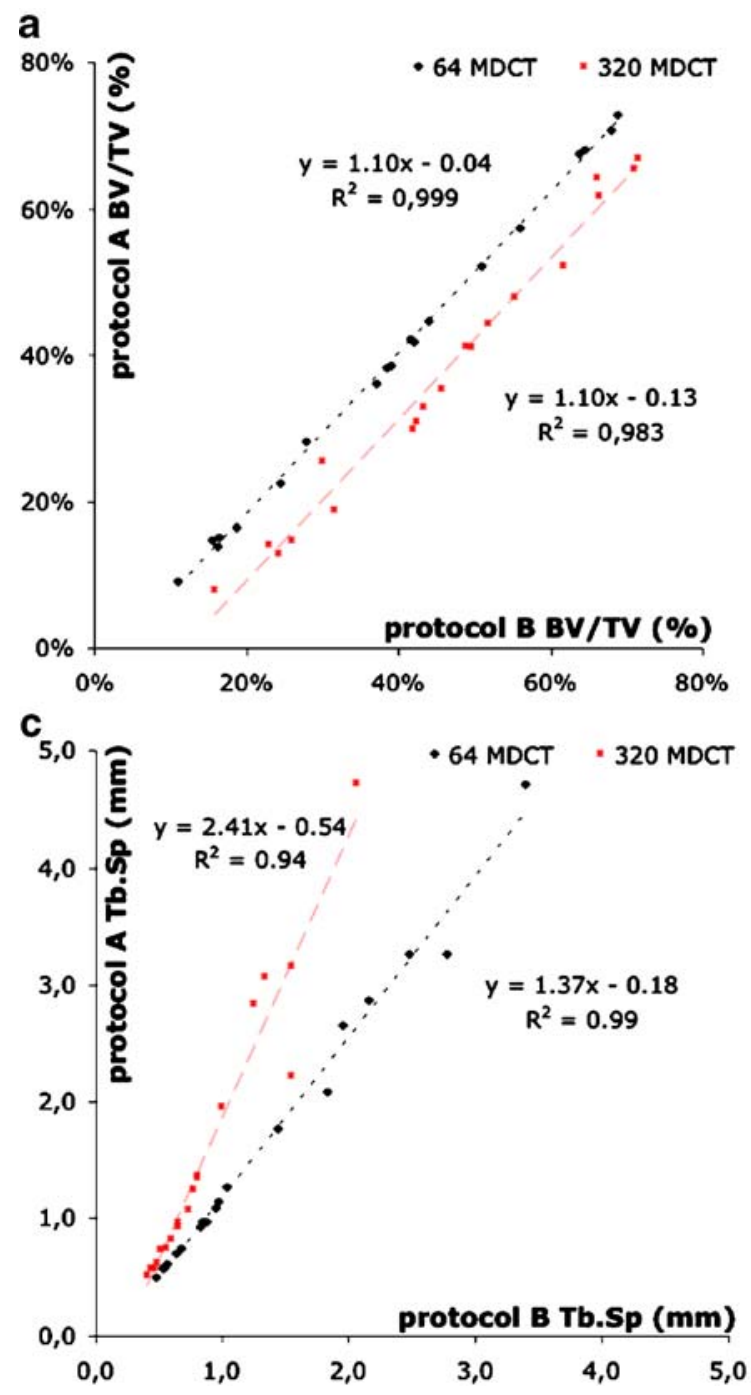

Fig. 5 Comparison of apparent trabecular structure measures (BV/ TV (a), Tb.N (b), Tb.Sp (c), Tb.Th (d)) derived using protocol A $(120 \mathrm{kVp}$ and $200 \mathrm{~mA})$ vs. protocol $\mathrm{B}(135 \mathrm{kVp}$ and $400 \mathrm{~mA})$ as

significant differences in absolute structure values. Aside from HR-MRI, high-resolution MDCT is an alternative imaging technique that has been used for in vivo trabecular bone imaging, but mainly for central sites such as the spine $[17,18,28]$. The aim of this study was to compare HRpQCT trabecular bone structure measures of the distal radius (at an isotropic resolution of $41 \mu \mathrm{m}$ ) with MDCT-derived structure measures. The study design used is unique in two aspects: to simulate a clinical, in vivo-like imaging setup, paraformaldehyde-fixed human cadaver distal forearms with intact surrounding soft tissue-were used. Furthermore, in this study the assessment of trabecular bone structure was evaluated using a 320-slice MDCT system and compared with a 64-slice MDCT system.

The "volume scan mode" of the 320-slice MDCT system offers a single rotation acquisition of $16 \mathrm{~cm}$ in the $z$ direction, thus avoiding interslice stitching artefacts and the

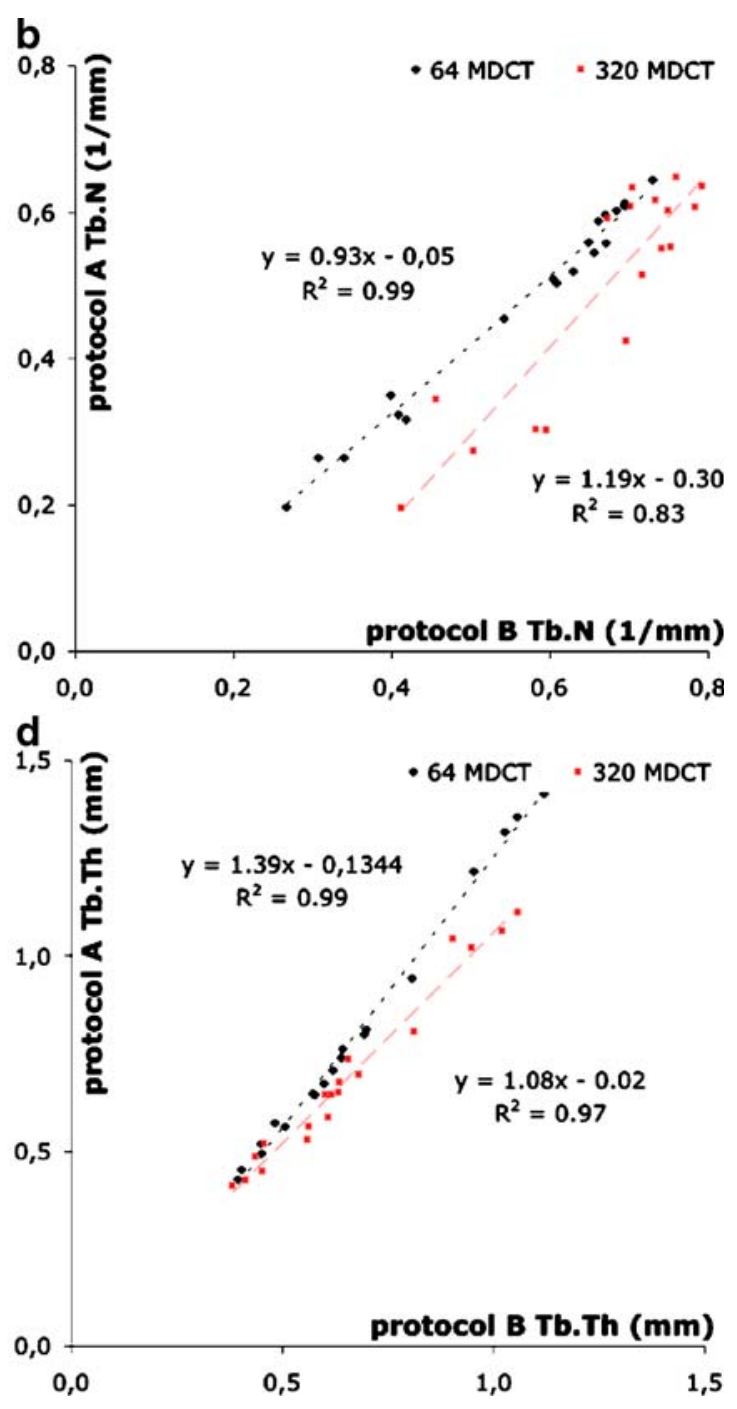

assessed with the 64-slice (black dots) and the 320-slice (red dots) MDCT device. Coefficient of determination $R^{2}$ is given

necessity of data interpolations, as required for standard helical CT acquisitions. Moreover, a novel algorithm enabling image reconstruction with a slice increment of $250 \mu \mathrm{m}$ (vs. $300 \mu \mathrm{m}$ ) is implemented in the 320 -slice device. Thus, when covering an imaging range of $9 \mathrm{~mm}$, as in our study, the 320 slice device computes 36 axial images, compared with 30 axial images from the 64-slice device. Regardless of these minor technical variations the yielded voxel sizes - for both devices - remain above the dimension of individual trabeculae. Thus - in line with published literature [38-40] — our data validate the anticipated result that both device types are equally well suited for the derivation of primary apparent structure measures. No quantifiable difference with regard to trabecular bone depiction in the $z$-direction was found between the 64- and 320-slice systems.

Our study protocol consisted of two different dose applications: the standard dose protocol A used in our 
clinic for peripheral bone imaging $(120 \mathrm{kVp}$ and $200 \mathrm{~mA})$, and a high-dose protocol $\mathrm{B}(135 \mathrm{kVp}$ and $400 \mathrm{~mA}$ ). The aim was to evaluate whether high-dose levels improve trabecular bone structure depiction. However, our results show no statistically significant differences between primary measures acquired using protocol A and B. In a study on trabecular bone structure analysis of the calcaneus performed by Patel et al. [41] four different imaging protocols were applied. The authors report that structure measures assessed with $120 \mathrm{kVp}$ and $150 \mathrm{~mA}$ did not differ substantially from those obtained with $120 \mathrm{kVp}$ and $300 \mathrm{~mA}$. Although our protocols ranged within different dose levels, one may overall hypothesize that beyond a given dose, an increase of the same does not improve the assessment of trabecular bone structure using MDCT. The identification of this dose threshold for different skeletal regions may be of interest for future research.

As possible MDCT device- and protocol-dependent alterations have been addressed, comparisons of MDCTwith HR-pQCT-derived structure measures are to be discussed next. Overall, our results show highly significant $(p<0.0001)$ correlations between MDCT and HR-pQCT, especially for BV/TV $(r=0.95-0.96)$ and Tb.Sp $(r=0.92-$ 0.93 ). However, both measures show a substantial overestimation when assessed with MDCT. These results correspond well with previously published data. Diederichs et al. [42] performed a study in which HR-pQCT and MDCT structure measures of proximal femur specimens were compared. In this study, BV/TV and Tb.Sp were also the two structure measures that correlated best with HRpQCT. However, the level of significance reached $(p<$ $0.01)$ and the correlation coefficients $\left(\mathrm{BV} / \mathrm{TV} r_{\text {Pearson }}=\right.$ 0.87; $\mathrm{Tb} . \mathrm{Sp} r_{\text {Pearson }}=0.66$ ) were both lower than those in our study. This is most likely because of soft-tissueinduced constraints (central vs. peripheral sites) in trabecular bone assessment, as investigated by Bauer et al. [43] The average MDCT-derived app. Tb.Th in our study yielded two- to threefold higher mean values than the corresponding HR-pQCT measure. An overestimation of trabecular dimensions based on partial volume effects may be the reason for this. However, considering that only about $50 \%$ of the trabeculae were detected with MDCT, one may also draw the more obvious conclusion that only thicker trabeculae were depicted, and thinner trabeculae were missed. Overall, no significant correlation was found for MDCT- and HR-pQCT-derived Tb.Th measures. As a result of MDCT resolutions $\left(234 \times 234 \times 500 \mu \mathrm{m}^{3}\right)$ beyond the average trabecular size, this is not unexpected and has already been observed by other investigators [38, 42-44]. At this point, it needs to be carefully considered that given pixel and voxel sizes are not equivalent to the true spatial resolution of an MDCT device. The spatial resolution is a measure that quantifies the ability of an imaging system to differentiate two objects of varying density from each other, whereas the voxel size reflects the display resolution.
The modulation transfer function (MTF) is commonly used to describe the spatial resolution in CT imaging. Among others it depends on the used kernel, contrast resolution and dosage level. Thus, although it is possible to shrink the FOV to $6 \mathrm{~cm}$ and decrease the in-plane pixel size to $117 \times 117 \mu \mathrm{m}^{2}$ for radius imaging, substantial benefits for the derivation of structure measures remain hypothetical. Besides, full coverage of the density calibration phantom would have not been assured, consecutively resulting in an inaccurate threshold definition. Thresholding may be considered as the first crucial postprocessing step in trabecular bone structure analysis. Careful identification of the threshold level and subsequent image binarisation need to ensure that dense bones do not only consist of "on" pixels and osteoporotic bones do not only consist of "off" pixels. Different methods have been proposed for MDCT image binarisation, among which visual optimisation-based approaches have frequently been used [17, 41, 43]. However, in this study-to avoid operatordependent bias - a correlation-based threshold definition [42], with HR-pQCT structure measures as a reference, has been applied.

One limitation of our study is the used sample size, which may be regarded small as the 20 specimens were obtained from only ten body donors, resulting in a restricted distribution of BMD and trabecular structure. However, it needs to be considered that the availability of human cadaver specimens with intact surrounding soft tissue is generally constrained.

Another limitation of this study-in terms of its comparability with daily clinical practice-is that by using human cadaver specimens, no motion-induced artefacts compromised image quality. For the standard 3min HR-pQCT scans, it is known that patient movement is a significant source of error, commonly necessitating reimaging or study exclusion. In this context, the faster data acquisition for MDCT systems (with an imaging time frame of at most $1 \mathrm{~s}$ ) would be expected to considerably reduce the occurrence of motion artefacts.

In summary, this study evaluated the assessment of trabecular structure measures from a 320-slice MDCT device and compared those with structure measures obtained from a 64-slice MDCT device. Using intact human cadaver forearm specimens an in vivo-like study design for distal radius imaging was introduced. HRpQCT at an isotropic resolution of $41 \mu \mathrm{m}$ was used as the standard of reference. No device- (320- vs. 64-slice) or protocol-dependent $(120 \mathrm{kVp}$ and $200 \mathrm{~mA}$ vs. $135 \mathrm{kVp}$ and $400 \mathrm{~mA}$ ) differences were found. Although substantial differences in absolute values were observed, overall, most MDCT-derived structure measures correlated highly significantly with HR-pQCT measures. Thus, even though accurate depiction of individual trabeculae is not given, these findings still confirm that MDCT is capable of quantifying characteristics of the trabecular bone network in the radius. 
Acknowledgements This work was supported by funding from an Elsbeth Bonhoff Grant (Charité 89832 232).

Open Access This article is distributed under the terms of the Creative Commons Attribution Noncommercial License which permits any noncommercial use, distribution, and reproduction in any medium, provided the original author(s) and source are credited.

\section{References}

1. NIH Consensus Development Panel on Osteoporosis Prevention, Diagnosis, and Therapy (2001) Osteoporosis prevention, diagnosis, and therapy. JAMA 285:785-795

2. Beck TJ, Ruff CB, Warden KE, Scott WW Jr, Rao GU (1990) Predicting femoral neck strength from bone mineral data. A structural approach. Invest Radiol 25:6-18

3. Bell GH, Cuthbertson DP, Orr J (1941) Strength and size of bone in relation to calcium intake. J Physiol 100:299-317

4. Hansson T, Roos B, Nachemson A (1980) The bone mineral content and ultimate compressive strength of lumbar vertebrae. Spine 5:46-55

5. McBroom RJ, Hayes WC, Edwards WT, Goldberg RP, White AA 3rd (1985) Prediction of vertebral body compressive fracture using quantitative computed tomography. J Bone Joint Surg Am 67:1206-1214

6. Mosekilde L, Bentzen SM, Ortoft G, Jorgensen J (1989) The predictive value of quantitative computed tomography for vertebral body compressive strength and ash density. Bone 10:465-470

7. Mosekilde L, Mosekilde L, Danielsen CC (1987) Biomechanical competence of vertebral trabecular bone in relation to ash density and age in normal individuals. Bone 8:79-85

8. Ammann P, Rizzoli R (2003) Bone strength and its determinants. Osteoporos Int 14(Suppl 3):S13-S18

9. Kanis JA, Borgstrom F, De Laet C, Johansson H, Johnell O, Jonsson B, Oden A, Zethraeus N, Pfleger B, Khaltaev N (2005) Assessment of fracture risk. Osteoporos Int 16:581589

10. Schuit SC, van der Klift M, Weel AE, de Laet CE, Burger H, Seeman E, Hofman A, Uitterlinden AG, van Leeuwen JP, Pols HA (2004) Fracture incidence and association with bone mineral density in elderly men and women: the Rotterdam study. Bone 34:195-202
11. Taylor BC, Schreiner PJ, Stone KL Fink HA, Cummings SR, Nevitt MC, Bowman PJ, Ensrud KE (2004) Longterm prediction of incident hip fracture risk in elderly white women: study of osteoporotic fractures. J Am Geriatr Soc 52:1479-1486

12. Kleerekoper M, Villanueva AR, Stanciu J, Rao DS, Parfitt AM (1985) The role of three-dimensional trabecular microstructure in the pathogenesis of vertebral compression fractures. Calcif Tissue Int 37:594-597

13. Ulrich D, van Rietbergen B, Laib A, Ruegsegger P (1999) The ability of three-dimensional structural indices to reflect mechanical aspects of trabecular bone. Bone 25:55-60

14. van der Linden JC, Weinans H (2007) Effects of microarchitecture on bone strength. Curr Osteoporos Rep 5:56-61

15. Alberich-Bayarri A, Marti-Bonmati L, Sanz-Requena R, Belloch E, Moratal D (2008) In vivo trabecular bone morphologic and mechanical relationship using high-resolution 3-T MRI. AJR Am J Roentgenol 191:721-726

16. Chang G, Pakin SK, Schweitzer ME, Saha PK, Regatte RR (2008) Adaptations in trabecular bone microarchitecture in Olympic athletes determined by 7T MRI. J Magn Reson Imaging 27:1089-1095

17. Graeff C, Timm W, Nickelsen TN, Farrerons J, Marin F, Barker C, Gluer CC (2007) Monitoring teriparatide-associated changes in vertebral microstructure by high-resolution $\mathrm{CT}$ in vivo: results from the EUROFORS study. J Bone Miner Res 22:1426-1433

18. Ito M, Ikeda K, Nishiguchi M, Shindo H, Uetani M, Hosoi T, Orimo H (2005) Multi-detector row CT imaging of vertebral microstructure for evaluation of fracture risk. J Bone Miner Res 20:1828-1836

19. Boutroy S, Bouxsein ML, Munoz F, Delmas PD (2005) In vivo assessment of trabecular bone microarchitecture by high-resolution peripheral quantitative computed tomography. J Clin Endocrinol Metab 90:6508-6515

20. MacNeil JA, Boyd SK (2007) Accuracy of high-resolution peripheral quantitative computed tomography for measurement of bone quality. Med Eng Phys 29:1096-1105
21. Ridler TW (1978) Picture thresholding using an iterative selection method. IEEE Trans Syst Man Cybern 8:630632

22. Hildebrand T, Laib A, Muller R, Dequeker J, Ruegsegger P (1999) Direct three-dimensional morphometric analysis of human cancellous bone: microstructural data from spine, femur, iliac crest, and calcaneus. J Bone Miner Res 14:1167-1174

23. Majumdar S, Genant HK (1997) Assessment of trabecular structure using high resolution magnetic resonance imaging. Stud Health Technol Inform 40:81-96

24. Chavassieux P, Asser Karsdal M, Segovia-Silvestre T, Neutzsky-Wulff AV, Chapurlat R, Boivin G, Delmas PD (2008) Mechanisms of the anabolic effects of teriparatide on bone: insight from the treatment of a patient with pycnodysostosis. J Bone Miner Res 23:1076-1083

25. Beck TJ, Looker AC, Ruff CB, Sievanen H, Wahner HW (2000) Structural trends in the aging femoral neck and proximal shaft: analysis of the Third National Health and Nutrition Examination Survey dual-energy X-ray absorptiometry data. J Bone Miner Res 15:2297-2304

26. Ciarelli TE, Fyhrie DP, Schaffler MB, Goldstein SA (2000) Variations in three-dimensional cancellous bone architecture of the proximal femur in female hip fractures and in controls. J Bone Miner Res 15:32-40

27. Legrand E, Audran M, Guggenbuhl P, Levasseur R, Chales G, Basle MF, Chappard D (2007) Trabecular bone microarchitecture is related to the number of risk factors and etiology in osteoporotic men. Microsc Res Tech 70:952-959

28. Chevalier F, Laval-Jeantet AM, LavalJeantet M, Bergot C (1992) CT image analysis of the vertebral trabecular network in vivo. Calcif Tissue Int 51:813

29. Durand EP, Ruegsegger P (1991) Cancellous bone structure: analysis of high-resolution CT images with the run-length method. J Comput Assist Tomogr 15:133-139 
30. Muller R, Hildebrand T, Ruegsegger P (1994) Non-invasive bone biopsy: a new method to analyse and display the three-dimensional structure of trabecular bone. Phys Med Biol 39:145-164

31. Sornay-Rendu E, Boutroy S, Munoz F, Delmas PD (2007) Alterations of cortical and trabecular architecture are associated with fractures in postmenopausal women, partially independent of decreased BMD measured by DXA: the OFELY study. J Bone Miner Res 22:425-433

32. Vico L, Zouch M, Amirouche A, Frere D, Laroche N, Koller B, Laib A, Thomas T, Alexandre C (2008) Highresolution pQCT analysis at the distal radius and tibia discriminates patients with recent wrist and femoral neck fractures. J Bone Miner Res 23:17411750

33. Johnell O, Kanis J (2005) Epidemiology of osteoporotic fractures. Osteoporos Int 16(Suppl 2):S3-S7

34. Barrett-Connor E, Sajjan SG, Siris ES, Miller PD, Chen YT, Markson LE (2008) Wrist fracture as a predictor of future fractures in younger versus older postmenopausal women: results from the National Osteoporosis Risk Assessment (NORA). Osteoporos Int 19:607-613
35. Kazakia GJ, Hyun B, Burghardt AJ, Krug R, Newitt DC, de Papp AE, Link TM, Majumdar S (2008) In vivo determination of bone structure in postmenopausal women: a comparison of HR-pQCT and high-field MR imaging. J Bone Miner Res 23:463-474

36. Krug R, Carballido-Gamio J, Banerjee S, Burghardt AJ, Link TM, Majumdar S (2008) In vivo ultra-high-field magnetic resonance imaging of trabecular bone microarchitecture at $7 \mathrm{~T}$. J Magn Reson Imaging 27:854-859

37. Krug R, Carballido-Gamio J, Burghardt AJ, Kazakia G, Hyun BH, Jobke B, Banerjee S, Huber M, Link TM, Majumdar S (2008) Assessment of trabecular bone structure comparing magnetic resonance imaging at 3 Tesla with high-resolution peripheral quantitative computed tomography ex vivo and in vivo. Osteoporos Int 19:653-661

38. Kothari M, Keaveny TM, Lin JC, Newitt DC, Genant HK, Majumdar S (1998) Impact of spatial resolution on the prediction of trabecular architecture parameters. Bone 22:437-443

39. Muller R, Koller B, Hildebrand T, Laib A, Gianolini S, Ruegsegger P (1996) Resolution dependency of microstructural properties of cancellous bone based on three-dimensional mu-tomography. Technol Health Care 4:113-119

40. Sode M, Burghardt AJ, Nissenson RA, Majumdar S (2008) Resolution dependence of the non-metric trabecular structure indices. Bone 42:728-736
41. Patel PV, Prevrhal S, Bauer JS, Phan C, Eckstein F, Lochmuller EM, Majumdar S, Link TM (2005) Trabecular bone structure obtained from multislice spiral computed tomography of the calcaneus predicts osteoporotic vertebral deformities. J Comput Assist Tomogr 29:246-253

42. Diederichs G, Link T, Marie K, Huber M, Rogalla P, Burghardt A, Majumdar S, Issever A (2008) Feasibility of measuring trabecular bone structure of the proximal femur using 64-slice multidetector computed tomography in a clinical setting. Calcif Tissue Int 83 (5):332-41

43. Bauer JS, Link TM, Burghardt A, Henning TD, Mueller D, Majumdar S, Prevrhal S (2007) Analysis of trabecular bone structure with multidetector spiral computed tomography in a simulated soft-tissue environment. Calcif Tissue Int 80:366-373

44. Bauer JS, Issever AS, Fischbeck M, Burghardt A, Eckstein F, Rummeny EJ, Majumdar S, Link TM (2004) Multislice-CT for structure analysis of trabecular bone-a comparison with micro-CT and biomechanical strength. Rofo 176:709-718 